\title{
Exploration of wild edible plants used by Gujjar and Bakerwal tribes of District Rajouri (J\&K), India
}

\section{R. Dangwal, Tajinder Singh* and Amandeep Singh}

Herbarium and Plant Systematic Lab., H.N.B. Garhwal Central University, S.R.T. Campus, Badshahithaul, Tehri-Garhwal-249199 (Uttarakhand), INDIA

*Corresponding author. E-mail:tajkhalsa@gmail.com

Received: January 2, 2014; Revised received: April 15, 2014; Accepted: April 25, 2014

Abstract: The analysis of wild plant exploration was carried out to document the wild edible plants of district Rajouri $(\mathrm{J} \& \mathrm{~K})$, India. The present investigation of wild edible plants were based on extensive and intensive field survey during 2009- 2011. A total of 58 wild edible plant species belonging to 50 genera and 39 families have been reported from the area. Rosaceae was the dominant family that represented 7 taxa, while Brassiceae, Fabaceae, Moraceae and Poaceae represented with 3 taxa each and other 33 families have less representation. Tree make up was the highest proportion of the edible species $28(41 \%)$ followed by 26 were herbs (38\%) and 14 were shrubs $(21 \%)$. Based on the requirements/edibility, majority of the plant species (26 species) are commonly used as fruits, 14 serve as vegetables, 1 species as flavoring agents (spices), roots, tubers of 3 species are eaten as raw and seeds/grains from 8 species for various substitute of food, whereas 2 species are used in making special drinks.

Keywords: District Rajouri, Gujjar and Bakarwal tribe, Wild edible plants

\section{INTRODUCTION}

Wild plants play a very vital role in the livelihoods of tribal communities. Wild resources provide materials for utensils and construction, and contribute to improved diets and health, food security, income generation and genetic experimentation (Kumar and Hamal, 2009). In developing countries, rural population who mainly comprise of herders, shepherds or other economically marginalized sections of the population use forests for grazing, firewood collection and numerous other subsistence needs (Kothari et al., 1989; Van Schaik et al., 1997; Saberwal and Ranagarajan, 2003). Understanding the local people's indigenous knowledge in biodiversity/resource management is one of the key issues for the development in present times (Kunwar and Duwadee, 2003).

Wild edible plants are major source of food for tribal inhabitants in forests. Edible parts of wild plants (fruits, flowers, leaves, tubers, inflorescence, roots, tubers, rhizome, etc.) are the nature's gift to mankind; these are not only delicious and refreshing but also the chief source of vitamins, minerals and proteins (Kumar and Hamal, 2009). About 1,000 species of these plants provide sustenance to tribal inhabitants in India (Ravikiran, 2008). In many parts of the world, wild edible plants are reported from forest, designated for extractive resources and managed by local communities for their own and other purposes (Jadhav et al., 2011). Food plants serve as alternatives to staple food during the period of food deficit are a valuable supplement for a nutritionally balanced diet and also one of the primary alternative sources of income for many rural and tribal communities (Shrestha and Dhillion, 2006).

The popularity of the wild forms of fruits, flowers and tubers declining continuously due to increasing of their demands for other food products, hence resulted the degradation of traditional knowledge, and it has been considered that, special attention should be paid to these wild food plants, which play a very important role in the livelihoods of the rural as well tribal communities (Jadhav et al., 2011).

Earlier, plants and their uses have been explored by several plant explorers in western Himalaya (Kachroo and Nahvi, 1976; Gupta et al., 1982; Kaul et al., 1987; Jain, 1991; Anonmyous, 1994; Lal et al., 1996; Maheswari, 2000; Rashid et al., 2008 and Dangwal et al., 2011, 2012a, 2012b). The present study help us to documents the wild relative of the plants. It provides information about valuable plants on which the tribal community depends. It also provides crop improvement due to their rich nutrient value. It also provides information about nutrient rich diet for tribal and landless communities. The study also helps for the development of silviculture practices in barren area and roadsides for approaching to drought conditions and in food deficient. Keeping above in view the main focus of the present study was to document the some wild edible plants of the district Rajouri used by Gujjar and Bakarwal tribes.

\section{MATERIALS AND METHODS}

Study area: District Rajouri is one of the district of 
J\&K having rich plant diversity with different topography and climate reaches up to alpine zones. It lies in western circle of Jammu division and bounded by district Poonch in North, district Reasi in West and (Mirpur) Pakistan in South and East. It lies in $30^{\circ}-50^{\prime}$ $\mathrm{N}$ to $33^{\circ}-30^{\prime} \mathrm{N}$ and $74^{0} \mathrm{E}$ to $74^{0}-10^{\prime} \mathrm{E}$. with an altitudinal range from $370-6000 \mathrm{~m}$ above sea level. The total forest area of district Rajouri is 1,267 sq. kms. During the present study, Nowshera and Budhal blocks were selected for the study. Nowshera block $\left(33^{\circ}-10^{\prime} \mathrm{N}\right.$ and $74^{0}-18^{\prime} \mathrm{E}$ ) was lies at elevation of $470 \mathrm{~m}-1200 \mathrm{~m}$ above sea level, covering an area of 482 sq. $\mathrm{kms}$ and Budhal block $\left(33^{\circ}-23^{\prime} \mathrm{N}\right.$ and $\left.74^{\circ}-41^{\prime} \mathrm{E}\right)$ was lies at elevation of $1000 \mathrm{~m}-4660 \mathrm{~m}$ above sea level and covering an area of 477 sq. kms.

Gujjar and Bakarwal tribes constitute the major segment of the district population and tribal race of J\&K state leading the nomadic life who graze their herds of sheep, goat and cattle from south of Pir Panjal range to Alpine pasture of the greater Himalaya in north, using mainly forest resources to fulfill their needs like food, fodder, forage, shelter, fuel wood, fiber and medicines etc. In winter season, they migrate from alpine pasture to subtropical zone of the district (Kalakote, Nowshera, Sunderbani) and in summer season they migrate to alpine zone of Pir Panjal range. The primary occupation of the tribes is livestock and secondary forest product on which they depend for food and fodder.

The extensive and intensive field survey of the slected blocks was made during 2009 to 2011 based on seasonal migration of Gujjar and Bakarwal tribes. During the course of the study, 5 different sites were selected (i.e. Nowshera and Lam in Nowshera block and Khawas, Koteranka and Budhal in Budhal block). Frequently field trips were made twice a month in each site of the blocks. Questionnaire was made for the documentation of information of plants being used as

Table. 1. List of wild edible plants and their parts used as food.

\begin{tabular}{|c|c|c|c|c|c|c|}
\hline $\begin{array}{l}\text { S. } \\
\text { N. }\end{array}$ & Family & Botanical name & Habit & $\begin{array}{l}\text { Local } \\
\text { name }\end{array}$ & $\begin{array}{l}\text { Flowering and } \\
\text { fruiting }\end{array}$ & Part used as food \\
\hline 1 & Acanthaceae & $\begin{array}{l}\text { Pteracanthus alatus } \\
\text { (Clarke) }\end{array}$ & Herb & - & $\begin{array}{l}\text { Fl.: Aug.-Oct. } \\
\text { Fr.: Oct.-Nov. }\end{array}$ & $\begin{array}{l}\text { Leaves and flowers } \\
\text { occasionally cooked } \\
\text { as vegetable. }\end{array}$ \\
\hline \multirow[t]{2}{*}{2} & Amaranthaceae & $\begin{array}{l}\text { Amaranthus viridis } \\
\text { L. }\end{array}$ & Herb & $\begin{array}{l}\text { Jungli } \\
\text { Ganaer }\end{array}$ & $\begin{array}{l}\text { Fl. \& Fr.: Jan.- } \\
\text { Dec. }\end{array}$ & $\begin{array}{l}\text { Young shoots and } \\
\text { leaves cooked as } \\
\text { vegetable. }\end{array}$ \\
\hline & & $\begin{array}{l}\text { Celosia argentea } \\
\text { Moq. in DC }\end{array}$ & Herb & - & $\begin{array}{l}\text { Fl. \& Fr.: Aug.- } \\
\text { Dec }\end{array}$ & $\begin{array}{l}\text { Leaves cooked as } \\
\text { vegetable }\end{array}$ \\
\hline 3 & Anacardiaceae & $\begin{array}{l}\text { Rhus parviflora } \\
\text { Roxb. }\end{array}$ & $\begin{array}{l}\text { Shrub } \\
\text { or } \\
\text { small } \\
\text { tree }\end{array}$ & Kakco & $\begin{array}{l}\text { Fl.: May-Jun. } \\
\text { Fr.: Jul.-Nov }\end{array}$ & Fruits are edible. \\
\hline 4 & Apocynaceae & $\begin{array}{l}\text { Carissa opaca } \\
\text { Stapy ex.Hains. }\end{array}$ & Shrub & Granda & $\begin{array}{l}\text { Fl. \& Fr. Aug.- } \\
\text { Dec }\end{array}$ & Fruits are edible \\
\hline 5 & Asteraceae & $\begin{array}{l}\text { Taraxacum } \\
\text { officinale Weber }\end{array}$ & Herb & Hand & $\begin{array}{l}\text { Fl. \& Fr: Feb.- } \\
\text { Oct. }\end{array}$ & Cooked as vegetable \\
\hline 6 & Balsaminaceae & $\begin{array}{l}\text { Impatiens balsa- } \\
\text { mina Linn. }\end{array}$ & Herb & Boti & Fl. \& Fr.: Jul.-Oct. & $\begin{array}{l}\text { Seed are edible, used } \\
\text { as condiments }\end{array}$ \\
\hline & & $\begin{array}{l}\text { Impatiens sulcata } \\
\text { Wallich in Roxb. }\end{array}$ & Herb & - & $\begin{array}{l}\text { Fl.: Jul.-Sept. } \\
\text { Fr.: Sept.-Oct. }\end{array}$ & Seeds are edible. \\
\hline 7 & Berberidacaea & $\begin{array}{l}\text { Berberis lycium } \\
\text { Royle in Trans. } \\
\text { Linn. Soc. }\end{array}$ & Shrub & $\begin{array}{l}\text { Simblu or } \\
\text { krumble }\end{array}$ & $\begin{array}{l}\text { Fl.: Apr.-Jun. } \\
\text { Fr.: Aug.-Sept. }\end{array}$ & $\begin{array}{l}\text { Ripe fruits edible, } \\
\text { also made into sauce. }\end{array}$ \\
\hline \multirow[t]{2}{*}{8} & Brassicaceae & $\begin{array}{l}\text { Lepidium sativum } \\
\text { L. }\end{array}$ & Herb & Sag & $\begin{array}{l}\text { Fl.: Feb.-Apr. } \\
\text { Fr.: Apr.-May }\end{array}$ & $\begin{array}{l}\text { Young leaves or } \\
\text { plant cooked as vege- } \\
\text { table. }\end{array}$ \\
\hline & & $\begin{array}{l}\text { Lepidium virgini- } \\
\text { cum L. }\end{array}$ & Herb & Sag & $\begin{array}{l}\text { Fl.: Feb.-Apr. } \\
\text { Fr.: Apr.-May }\end{array}$ & $\begin{array}{l}\text { Young shoot occa- } \\
\text { sionally taken as } \\
\text { vegetable }\end{array}$ \\
\hline 9 & $\begin{array}{l}\text { Caesalpini- } \\
\text { aceae }\end{array}$ & $\begin{array}{l}\text { Bauhinia variegata } \\
\text { L. }\end{array}$ & Tree & - & $\begin{array}{l}\text { Fl.: Feb.-Apr. } \\
\text { Fr.: May-Aug. }\end{array}$ & $\begin{array}{l}\text { Young flowers eaten } \\
\text { as vegetable. }\end{array}$ \\
\hline 10 & Caprifoliaceae & $\begin{array}{l}\text { Viburnum grandi- } \\
\text { florum Wallich ec } \\
\text { DC. }\end{array}$ & Shrub & Gucjh & $\begin{array}{l}\text { Fl.: Mar.-May } \\
\text { Fr. \& Fr.: Aug.- } \\
\text { Oct. }\end{array}$ & Fruits are edible \\
\hline
\end{tabular}




\begin{tabular}{|c|c|c|c|c|c|c|}
\hline $\begin{array}{l}\text { S. } \\
\text { N. }\end{array}$ & Family & Botanical name & Habit & $\begin{array}{l}\text { Local } \\
\text { name }\end{array}$ & $\begin{array}{l}\text { Flowering } \\
\text { and fruiting }\end{array}$ & Part used as food \\
\hline 11 & Combretaceae & $\begin{array}{l}\text { Terminalia bellirica } \\
\text { (Gaertner) Roxb., }\end{array}$ & Tree & Rheed & $\begin{array}{l}\text { Fl.: Apr.-Jun. } \\
\text { Fr.: Jun.-Jul. }\end{array}$ & Fruits kernels edible. \\
\hline 12 & Cucurbitaceae & $\begin{array}{l}\text { Coccinia grandis } \\
\text { (L.) Voigt. }\end{array}$ & Herb & $\begin{array}{l}\text { Jangli } \\
\text { Kakhdi } \\
\text { or khera }\end{array}$ & $\begin{array}{l}\text { Fl.: Jun.-Jul. } \\
\text { Fr.: Aug.-Oct. }\end{array}$ & Fruits are cooked as vegetable. \\
\hline 13 & Dioscoreaceae & $\begin{array}{l}\text { Dioscorea bulbifera } \\
\text { L. }\end{array}$ & Herb & - & $\begin{array}{l}\text { Fl.: Jul.-Nov. } \\
\text { Fr.: Oct.-Jan. }\end{array}$ & $\begin{array}{l}\text { Tubers are edible as well as } \\
\text { medicinal, much liked by wild } \\
\text { life. }\end{array}$ \\
\hline 14 & Elaeagnaceae & $\begin{array}{l}\text { Eleagnus parvifolia } \\
\text { wallich ex Royle. }\end{array}$ & Shrub & - & $\begin{array}{l}\text { Fl.: Mar.-Apr. } \\
\text { Fr.: Jun.-Sept. }\end{array}$ & Fruits are edible \\
\hline 15 & Ericaceae & $\begin{array}{l}\text { Rhododendron } \\
\text { arboreum Smith }\end{array}$ & Tree & Harduli & $\begin{array}{l}\text { Fl.: Mar.-May } \\
\text { Fr. Apr.-Nov. }\end{array}$ & $\begin{array}{l}\text { Flowers eaten raw or made into } \\
\text { sauce, jellies, jams or refresh- } \\
\text { ing drinks. }\end{array}$ \\
\hline 16 & Euphorbiaceae & $\begin{array}{l}\text { Phyllanthus } \\
\text { emblica L. }\end{array}$ & Tree & Ambla & $\begin{array}{l}\text { Fl.: Feb.-Apr. } \\
\text { Fr.: Sept.- } \\
\text { Nov. }\end{array}$ & $\begin{array}{l}\text { Fruits eaten as raw and also } \\
\text { made Pickles from fruits }\end{array}$ \\
\hline \multirow[t]{3}{*}{17} & Fabaceae & $\begin{array}{l}\text { Indigofera heteran- } \\
\text { tha Wallich ex Bran- } \\
\text { dis }\end{array}$ & Shrub & - & $\begin{array}{l}\text { Fl.: May-Aug. } \\
\text { Fr.: Sept.- } \\
\text { Nov. }\end{array}$ & $\begin{array}{l}\text { Flower used as } \\
\text { vegetable. }\end{array}$ \\
\hline & & Vigna vexillata $\mathrm{L}$. & Herb & $\begin{array}{l}\text { Jangli } \\
\text { Dal }\end{array}$ & $\begin{array}{l}\text { Fl. \& Fr.: } \\
\text { Aug.-Nov. }\end{array}$ & $\begin{array}{l}\text { Fusiform root edible, some- } \\
\text { times seeds also }\end{array}$ \\
\hline & & $\begin{array}{l}\text { Quercus leucotricho- } \\
\text { phora A. Camus in } \\
\text { Riviera Sci. }\end{array}$ & Tree & Rhain & $\begin{array}{l}\text { Fl.: Mar.-Apr. } \\
\text { Fr.: Oct.-Jan. }\end{array}$ & $\begin{array}{l}\text { Fruits are eaten by monkeys } \\
\text { and bears and some time by } \\
\text { tribal people also. }\end{array}$ \\
\hline 18 & Flacourtiaceae & $\begin{array}{l}\text { Flacourtia indica } \\
\text { (Burm.f.) }\end{array}$ & Tree & - & $\begin{array}{l}\text { Fl.: Feb.-Mar. } \\
\text { Fr.; Apr.-Jun. }\end{array}$ & Fruits are edible. \\
\hline 19 & $\begin{array}{l}\text { Hippocastana- } \\
\text { ceae }\end{array}$ & $\begin{array}{l}\text { Aesculus indica } \\
\text { (Coler. ex Cambess) } \\
\text { Hook. in Curtis }\end{array}$ & Tree & Bankhori & $\begin{array}{l}\text { Fl.: Mar.-Apr. } \\
\text { Fr.: Jul.-Aug. }\end{array}$ & $\begin{array}{l}\text { Sub-acid pulp (aril) of fruit is } \\
\text { edible. }\end{array}$ \\
\hline 20 & Lamiaceae & $\begin{array}{l}\text { Leucus lanata Benth. } \\
\text { in Wallich }\end{array}$ & Herb & - & $\begin{array}{l}\text { Fl.\& Fr.: Al- } \\
\text { most through- } \\
\text { out the year }\end{array}$ & $\begin{array}{l}\text { Young shoots cooked as vege- } \\
\text { table. }\end{array}$ \\
\hline \multirow[t]{3}{*}{21} & Moraceae & $\begin{array}{l}\text { Ficus auriculata } \\
\text { Lour. }\end{array}$ & Tree & Tose & $\begin{array}{l}\text { Fl.: Mar.-May } \\
\text { Fr.: Jun.-Jul. }\end{array}$ & $\begin{array}{l}\text { Fruits are edible when ripe and } \\
\text { unripe fruits are used as vegeta- } \\
\text { ble. }\end{array}$ \\
\hline & & Ficus palmata Forsk. & Tree & Fagwara & $\begin{array}{l}\text { Fr. \& Fr.: Jun. } \\
\text {-Aug. }\end{array}$ & $\begin{array}{l}\text { Fruits delicious in taste, often } \\
\text { taken raw with salt or filled in- } \\
\text { side the bread. }\end{array}$ \\
\hline & & $\begin{array}{l}\text { Ficus semicordata } \\
\text { Buch.-Ham.ex J. E. } \\
\text { Smith in Rees. }\end{array}$ & Tree & - & $\begin{array}{l}\text { Fl.: May- Jun. } \\
\text { Fr.: Jun.-Oct. }\end{array}$ & Fruits edible. \\
\hline 22 & Myrtaceae & $\begin{array}{l}\text { Syzygium cumini (L.) } \\
\text { Skeels }\end{array}$ & Tree & Jamun & $\begin{array}{l}\text { Fl.: Mar.-May } \\
\text { Fr.: Jun.-Jul. }\end{array}$ & Ripe fruits are edible. \\
\hline 23 & Nyctaginaceae & Boerhavia diffusa $\mathrm{L}$. & Herb & Bal kakra & $\begin{array}{l}\text { Fl. \& Fr.: } \\
\text { Aug.-Dec. }\end{array}$ & $\begin{array}{l}\text { Leaves and tender shoots occa- } \\
\text { sionally made into vegetable; } \\
\text { roots Chewed as energy tonic }\end{array}$ \\
\hline 24 & Oleaceae & $\begin{array}{l}\text { Olea ferruginea } \\
\text { Royle }\end{array}$ & Tree & Kaaw & $\begin{array}{l}\text { Fl.: Feb.-Apr. } \\
\text { Fr.: Oct.-Nov. }\end{array}$ & Fruits edible. \\
\hline 25 & Oxalidaceae & Oxalis corniculata $\mathrm{L}$. & Herb & Kathimili & $\begin{array}{l}\text { Fl. \& Fr.: Al- } \\
\text { most through- } \\
\text { out the year }\end{array}$ & $\begin{array}{l}\text { Leaves taken as salad or } \\
\text { cooked as vegetable. }\end{array}$ \\
\hline \multirow[t]{2}{*}{26} & Pinaceae & $\begin{array}{l}\text { Picea smithiana } \\
\text { (Wallich) Boisser. }\end{array}$ & Tree & - & $\begin{array}{l}\text { Fl. \& Fr.: } \\
\text { Sept.-Dec. }\end{array}$ & Seeds are edible \\
\hline & & $\begin{array}{l}\text { Pinus roxburghii } \\
\text { Sargent }\end{array}$ & Tree & Chir & $\begin{array}{l}\text { Fl. \& Fr.: Mar. } \\
\text {-Jun. }\end{array}$ & Seeds are edible. \\
\hline \multirow[t]{3}{*}{27} & Poaceae & $\begin{array}{l}\text { Echinochloa colona } \\
\text { (L.) Link. }\end{array}$ & Herb & Gass & $\begin{array}{l}\text { Fl. \& Fr.: Jul.- } \\
\text { Oct. }\end{array}$ & $\begin{array}{l}\text { Grains are edible, prepared as } \\
\text { rice. }\end{array}$ \\
\hline & & $\begin{array}{l}\text { Setaria glauca }(\mathrm{L} .) \\
\text { P. Beav. }\end{array}$ & Herb & Gass & $\begin{array}{l}\text { Fl. \& Fr.: } \\
\text { Aug.-Nov. }\end{array}$ & $\begin{array}{l}\text { Grains occasionally eaten, often } \\
\text { used in local beverages. }\end{array}$ \\
\hline & & $\begin{array}{l}\text { Setaria viridis (L.) P. } \\
\text { Beauv. }\end{array}$ & Herb & Gass & $\begin{array}{l}\text { Fl. \& Fr.: } \\
\text { Aug.-Dec. }\end{array}$ & The grains are edible. \\
\hline
\end{tabular}




\begin{tabular}{|c|c|c|c|c|c|c|}
\hline $\begin{array}{l}\text { S. } \\
\text { N. }\end{array}$ & Family & Botanical name & Habit & $\begin{array}{l}\text { Local } \\
\text { name }\end{array}$ & $\begin{array}{l}\text { Flowering } \\
\text { and fruiting }\end{array}$ & Part used as food \\
\hline \multirow[t]{2}{*}{28} & Polygonaceae & $\begin{array}{l}\text { Rumex nepalensis } \\
\text { Sprengel }\end{array}$ & Herb & Hebli & $\begin{array}{l}\text { Fl.: Apr.-Aug. } \\
\text { Fr.: Aug.-Oct. }\end{array}$ & $\begin{array}{l}\text { Leaves cooked as } \\
\text { vegetables }\end{array}$ \\
\hline & & $\begin{array}{l}\text { Fagopyrum dibotrys } \mathrm{D} . \\
\text { Don. }\end{array}$ & Herb & Sag ka & $\begin{array}{l}\text { Fl.: Jul.-Sept. } \\
\text { Fr.: Sept.-Nov. }\end{array}$ & $\begin{array}{l}\text { Occasionally used as } \\
\text { vegetable. }\end{array}$ \\
\hline 29 & Puniceae & Punica granatum $\mathrm{L}$. & $\begin{array}{l}\text { Shrub or } \\
\text { small tree }\end{array}$ & Durni. & $\begin{array}{l}\text { Fl.: Apr.-Jun. } \\
\text { Fr.: Jul.-Dec. }\end{array}$ & $\begin{array}{l}\text { Fruits are edible, wild } \\
\text { ones sour, made into } \\
\text { sauce or chatney. }\end{array}$ \\
\hline \multirow[t]{2}{*}{30} & Rhamnaceae & $\begin{array}{l}\text { Ziziphus glaberrima } \\
\text { (Sedgwick) }\end{array}$ & $\begin{array}{l}\text { Shrub or } \\
\text { small tree }\end{array}$ & Bari & $\begin{array}{l}\text { Fl.: Sept.-Oct. } \\
\text { Fr.: Dec.-Feb. }\end{array}$ & Fruits are edible. \\
\hline & & $\begin{array}{l}\text { Ziziphus maurtiana } \\
\text { Lam. }\end{array}$ & $\begin{array}{l}\text { Shrub or } \\
\text { small tree }\end{array}$ & Baari & $\begin{array}{l}\text { Fl.: Jun.-Aug. } \\
\text { Fr.: Feb.-Mar. }\end{array}$ & Fruits are edible. \\
\hline \multirow[t]{7}{*}{31} & Rosaceae & $\begin{array}{l}\text { Duchesnea indica } \\
\text { (Andrews)Focke in } \\
\text { Engler. }\end{array}$ & Herb & $\begin{array}{l}\text { Jangli } \\
\text { akare. }\end{array}$ & $\begin{array}{l}\text { Fl.: Mar.-Jul. } \\
\text { Fr.: Oct.-Apr. }\end{array}$ & $\begin{array}{l}\text { Fruits are edible but } \\
\text { not much tasty, taken } \\
\text { by wild life. }\end{array}$ \\
\hline & & $\begin{array}{l}\text { Fragaria nubicola } \\
\text { Lindley ex Lacaita in J. } \\
\text { Linn }\end{array}$ & Herb & Mava & $\begin{array}{l}\text { Fl.: Mar.-Apr. } \\
\text { Fr.: Jun.-Jul. }\end{array}$ & Fruits are edible. \\
\hline & & Prinsepia utilis Royle & Shrub & Burkhui & $\begin{array}{l}\text { Fl.: Feb.-Mar. } \\
\text { Fr.: May-Jun. }\end{array}$ & $\begin{array}{l}\text { Seeds yield oil, used as } \\
\text { edible purposes. } \\
\text { Fruits are edible }\end{array}$ \\
\hline & & $\begin{array}{l}\text { Pyrus pashia Buch.- } \\
\text { Ham. ex D.Don. }\end{array}$ & Tree & Kathari & $\begin{array}{l}\text { Fl.: Feb.-Mar. } \\
\text { Fr.: May-Dec. }\end{array}$ & Ripe fruits are edible. \\
\hline & & $\begin{array}{l}\text { Rubus ellipticus Smith } \\
\text { in Rees. }\end{array}$ & Shrub & Akhare & $\begin{array}{l}\text { Fl.: Mar.-Apr. } \\
\text { Fr.: May-Jun. }\end{array}$ & Fruits are edible. \\
\hline & & $\begin{array}{l}\text { Rubus macilentus Cam- } \\
\text { bess. in Jacquem. }\end{array}$ & Shrub & $\begin{array}{l}\text { Kale } \\
\text { Akhare }\end{array}$ & $\begin{array}{l}\text { Fl.: Mar.-May } \\
\text { Fr.: Jun.-Sept. }\end{array}$ & Fruits are edible. \\
\hline & & Rubus niveus Thumb. & Shrub & Akhare & $\begin{array}{l}\text { Fl. \& Fr.: Feb.- } \\
\text { Aug. }\end{array}$ & Fruits are edible. \\
\hline 32 & Sapindaceae & $\begin{array}{l}\text { Dodonea angustifolia } \\
\text { L. }\end{array}$ & Shrub & Snatha & $\begin{array}{l}\text { Fl.: Aug.-Nov. } \\
\text { Fr.: Oct.-Jan. }\end{array}$ & $\begin{array}{l}\text { Seeds occasionally } \\
\text { edible, liked by mon- } \\
\text { key. }\end{array}$ \\
\hline \multirow[t]{2}{*}{33} & Smilacaceae & Smilax aspera $\mathrm{L}$. & $\begin{array}{l}\text { Climber } \\
\text { shrub }\end{array}$ & - & $\begin{array}{l}\text { Fl.: Jun.-Nov. } \\
\text { Fr.: Sept.-Dec. }\end{array}$ & $\begin{array}{l}\text { Occasionally young- } \\
\text { leaves are cooked. }\end{array}$ \\
\hline & & Smilax parviflora Wall & $\begin{array}{l}\text { Climber } \\
\text { shrub }\end{array}$ & - & Fl.: Apr.-Jun. & $\begin{array}{l}\text { Occasionally young } \\
\text { shoots used as vegeta- } \\
\text { ble. }\end{array}$ \\
\hline 34 & Solanaceae & Solanum nigrum $\mathrm{L}$. & Herb & $\begin{array}{l}\text { Kach } \\
\text { mach }\end{array}$ & $\begin{array}{l}\text { Fl. \& Fr.: Al- } \\
\text { most through- } \\
\text { out the year }\end{array}$ & Fruits are edible. \\
\hline 35 & Tiliaceae & $\begin{array}{l}\text { Grewia opitva J.R } \\
\text { Drummond ex Burret in } \\
\text { Notizbl. }\end{array}$ & Tree & $\begin{array}{l}\text { Thaman } \\
\text { or Dha- } \\
\text { man }\end{array}$ & $\begin{array}{l}\text { Fl.: Apr.-Jun. } \\
\text { Fr.: Aug.-Nov. }\end{array}$ & $\begin{array}{l}\text { Fruits are edible and } \\
\text { medicinal. }\end{array}$ \\
\hline 36 & Ulmaceae & Celtis eriocarpa Decne. & Tree & Kharik & $\begin{array}{l}\text { Fl.: Mar.-Apr. } \\
\text { Fr.: Sept.-Nov. }\end{array}$ & Fruits are edible. \\
\hline 37 & Urticaceae & Urtica dioica $\mathrm{L}$. & Herb & $\begin{array}{l}\text { Panayali } \\
\text { or Kinji }\end{array}$ & $\begin{array}{l}\text { Fl. \& Fr.: Aug. } \\
\text {-Apr. }\end{array}$ & $\begin{array}{l}\text { Young branches and } \\
\text { leaves used as deli- } \\
\text { cious pot herb, seed oil } \\
\text { is edible. }\end{array}$ \\
\hline 38 & Verbenaceae & $\begin{array}{l}\text { Callicarpa macrophylla } \\
\text { Vahal }\end{array}$ & Shrub & - & $\begin{array}{l}\text { Fl.: Jul.-Sept. } \\
\text { Fr.: Aug.-Oct. }\end{array}$ & Fruits edible. \\
\hline 39 & Vitaceae & $\begin{array}{l}\text { Ampelocissus rugosa } \\
\text { Wallich }\end{array}$ & $\begin{array}{l}\text { Climber } \\
\text { herb }\end{array}$ & - & $\begin{array}{l}\text { Fl.: Apr. - Jun. } \\
\text { Fr.: Sept.-Oct. }\end{array}$ & Ripe fruit is edible \\
\hline
\end{tabular}


food, their available vernacular names etc. from local inhabitants. The collected plants were identified with the help of published floras, literature and monographs i.e. Hooker (1906); Sharma and Kachroo (1983); Swami and Gupta (1998); Gaur (1999) and were confirmed from the authentic regional Herbaria at Botanical Survey of India, Northern Circle, Dehradun (BSD), Herbarium of Forest Research Institute (DD), Dehradun and HNB Garhwal University, Herbarium (GUH), Srinagar Garhwal, Uttarakhand and deposited in the Herbarium of Department of Botany, SRT Campus Badshahi Thaul, district Tehri Garhwal.

\section{RESULTS AND DISCUSSION}

During the present study, a total of 58 wild edible plants belonging to 50 genera and 39 families were reported (Table 1). Family Rosaceae was the dominant family representing 7 taxa, while Brassiceae, Fabaceae, Moraceae and Poaceae followed with 3 taxa each and other 33 families had less representation. Trees made up the highest proportion of the edible species 28 (41\%), followed by 26 herbs (38\%) and 14 shrubs $(21 \%)$.

Earlier, Rashid et al. (2008), have reported 57 wild edible plants belonging to 33 families from district Rajouri (J\&K), while Kumar and Hamal (2009) reported 50 wild edible plants belong to 33 families from Kishtwar high altitude National Park in northwest Himalaya. Jadhav et al. (2011) reported total 50 wild edible plants from Kolhapur district of Maharashtra. Based on the edibility/consumption, 26 wild plant species were used as fruit, 14 plant species as a vegetable, 1 species as flavoring agents (spices), roots as well as tubers of 3 species were eaten as raw, seeds/ grains of 8 species sometimes used as substitute of food, whereas 2 species were used in making special drinks.

Due to the remoteness of these tribal people, they are economically weak due lack of modern facilities. The use of wild edible plants can substantiate vitamins, proteins and fat contents in the human diet besides being an important source of cash earning to these tribes. The fruits of the plant species like Ficus palmata, Punica granatum, Phyllanthus emblica, Pyrus pashia, Rubus ellipticus, Syzygium cumini, Terminalia bellirica, Ziziphus mauritiana and leaves of Amaranthus viridis, Rumex nepalensis and flowers of Rhododendron arboreum are highly utilized by local people and are highly commercialized in the market. Due to over exploitation, overgrazing, urbanization etc. the status of Phyllanthus emblica, Punica granatum are at risk.

\section{Conclusion}

It was concluded that, the local inhabitants of the study area use 58 plants as wild food plants. These are not only delicious and refreshing but also rich in nutrients such as fats, carbohydrates and proteins. Due to their rich nutrient values most the tribal communities depend on these wild plants for their livelihood as well as commercial purposes. It is also noticed that due to over exploitation of forest recourses by them, these valuable plants are loosing their existence, so it is necessary to develop the socioeconomic as well as sustainable use these forest resources and also to develop agro-based and silvicultural practices in the forest so that these valuable gift of nature do not vanish from the area of $\mathrm{J} \& \mathrm{~K}$.

\section{REFERENCES}

Anonmyous (1994). Ethno-botany in India- A attains report (Ministry of Environment and Forests, Govt. of India).

Dangwal, L. R. and Singh, T. (2012a). Comparative vegetational analysis and Pinus roxburghii Sarg regeneration in relation to their disturbances in some Chirpine forest of Block Nowshera, district Rajouri, J\&K, India, ISCA J. Biological Sci., 1(1): 47-54.

Dangwal, L.R., Singh, A., Sharma A. and Singh, T. (2011). Diversity of weed species in wheat fields of block Nowshera District Rajouri (J\&K), Indian J. Weed Sc., 43(1\&2): 94-96.

Dangwal, L.R., Singh, T., Singh, A. and Sharma, A.(2012b). Species composition of woody plants in forest of Block Nowshera, District Rajouri (J\&K), India. International Journal of Current Research, 4 (5):5-10.

Gaur, R. D. (1999). Flora of district Garhwal: North-West Himalaya (with ethnobotanical Notes). Transmedia, Srinagar Garhwal.

Gupta, O.P., Srivastava, T.N., Gupta, S.C. and Badola, D.P. (1982). An ethnobotany and phytochemical screening of higher altitude plants of Ladakh Part II. Bull. Medico-Ethnobot. Res., 1: 301-317.

Hooker, J. D. (1906). A sketch of the Flora of British India, Oxford Publication.

Jadhav, V.D., Mahadkar, S.D. and Valvi, S.R. (2011). Documentation and ethnobotanical survey of wild edible plants from Kolapur district. Recent research in Science and Technology, 3(12):58-63.

Jain, S.K. (1991). Dictionary of Indian folk medicine and ethnobotany (Deep Publication, New Delhi).

Kachroo, P. and Navi, I.M. (1976). Ethno botany of Kashmiris forest flora of Srinagar and plants of Neighborhood Dehra Dun, India, pp. 239-263.

Kaul, M.K., Sharma, P.K. and Singh. V. (1987). Ethnobotanical studies in North-West and transHimalaya IV. Some traditionally tea substitutes from J\&K state. Himalayan Plant J., 4:23-28.

Kothari, A., Pande, P., Singh, S. and Variava, D. (1989). Management of National Parks and Wildlife Sanctuaries in India: A status report. Indian Institute of Public Adminstration, Delhi, India.

Kumar, S. and Hamal, I.A. (2009). Wild edibles of Kishtwar high altitude National Park in northwest Himalaya, Jammu \& Kashmir (India); Ethnobotanical Leaflet, 13: 195-202.

Kunwar, R. M. and Duwadee, N. P. S. (2003). Ethnobotanical notes on flora of Khaptad National Park, far-western Nepal. Himalayan Journal of Sciences, 1 (1):25-30.

Lal, B., Vats, S.K., Singh R.D. and Gupta, A.K. (1996). Plants used as ethnomedicine and supplement food by Gaddis of Himachal Pradesh, India, In: Jain S.K. (eds.) 
Ethnobiology in Human Welfare, New Delhi.

Maheswari, J.K. (2000). (Ed.) Ethno botany and medicinal plants of Indian Subcontinant, (Scientific Publishers, Jodhpur, pp. 672.

Rashid, A., Anand, V.K. and Serwar J. (2008). Less known wild edible plants used by the Gujjar Tribe of District Rajouri, Jammu \& Kashmir State, India; Int. J. Bot., 4 (2):219- 224.

Ravikiran, G. (2008). A taste of Wild- Undiscovered riches, The Hindu.

Saberwal, V. and Rangarajan, M. (2003). Battles over nature: Science and politics of conservation. NewDelhi, India.

Sharma, B.M. and Kachroo, P. (1983). Flora of Jammu and
Plants of neighborhood. Bishen Singh Mahendra Pal Singh, Dehradun.

Shrestha, P. M. and Dhillion, S. S. (2006). Diversity and traditional knowledge concerning wild food species in a locally managed forest in Nepal. Agroforestry Systems, 66:55-63.

Swami, A. and Gupta, B.K. (1998). Flora of Udhampur. Bishen Singh Mahendra Pal Singh, Dehradun, India.

Van Schaik;, C.P., Terborgh, J. and Dugelby, B. (1997). The silent crisis: the state of rain forest nature preserves. In: R. Kramer, C. van Schaik and J. Jonson, (eds.), Last stand: protected area and defence of tropical biodiversity, pp. 64-89. Oxford University Press, New York, USA. 